\title{
Comparison of the application value of two commonly used minimally invasive spinal surgery in the treatment of lumbar disc herniation
}

\author{
YINGBO ZHANG, JINPING CHEN, HAIYANG XIE, KUI LI, YE WANG, \\ QIAN CHEN, CHENG JIANG, JIANGTAO HE and NENGGAO FU
}

Department of Orthopaedics, Affiliated Hospital of North Sichuan Medical College, Nanchong, Sichuan 637000, P.R. China

Received June 26, 2020; Accepted November 25, 2020

DOI: $10.3892 / \mathrm{etm} .2021 .9730$

\begin{abstract}
The aim of the study was to compare the application value of percutaneous transforaminal endoscopic discectomy (PTED) and microendoscopic discectomy (MED) in the treatment of lumbar disc herniation (LDH). From January 2017 to July 2018, 108 LDH patients undergoing surgical treatment in our hospital were collected and divided into PTED group (treated with PTED, $\mathrm{n}=50$ ) and MED group (treated with MED, $n=58$ ). The operation parameter index level, complications, recurrence and pain score (VAS), Oswestry disability index (ODI) and Japanese Orthopaedic Association Scale (JOA) were compared between the two groups. VAS, ODI and JOA scores of the two groups were significantly decreased after operation $(\mathrm{P}<0.05)$, but there was no significant difference between the two groups $(\mathrm{P}>0.05)$. Complications and recurrence of the two groups were similar $(\mathrm{P}>0.05)$. MED was superior to PTED in the number of intraoperative fluoroscopy and operation time, while PTED was superior to MED in intraoperative blood loss, incision length, length of hospital stay and bed rest time $(\mathrm{P}<0.05)$. Both PTED and MED can effectively treat LDH. Referring to clinical data, PTED may be the first choice for LDH treatment.
\end{abstract}

\section{Introduction}

Lumbar disc herniation (LDH) is the most common spinal disease in clinic, and it is also the cause of pain and discomfort in waist and lower limbs (1). It is reported that about 9\% of individuals worldwide suffer from LDH (2). Conservative treatment and surgical treatment are mainly adopted clinically. After the failure of conservative treatment, surgery is the main

Correspondence to: $\mathrm{Mr}$ Yingbo Zhang, Department of Orthopaedics, Affiliated Hospital of North Sichuan Medical College, 1 Maoyuan South Road, Nanchong, Sichuan 637000, P.R. China E-mail: zrlbh3@163.com

Key words: lumbar disc herniation, percutaneous transforaminal endoscopic discectomy, microendoscopic discectomy treatment for $\mathrm{LDH}$ patients (3). In the past 10 years, due to the rapid development of medical devices and the strong demand of patients, spinal endoscopy has developed rapidly (4). At present, traditional open surgery has been gradually replaced by minimally invasive spinal surgery, which has the advantages of small incision, less pain, quick recovery, and short length of hospital stay (5).

Percutaneous transforaminal endoscopic discectomy (PTED) and microendoscopic discectomy (MED) are two common spinal endoscopic techniques that have been used in recent years. PTED is a combination of endoscopic technology and percutaneous puncture technology, which can establish a working channel under local anesthesia through the intervertebral foramen approach, the intervertebral space approach or the lateral approach of the intervertebral foramen to remove the protruding nucleus (6). PTED, as a common surgical method in minimally invasive spine surgery, has the advantages of being minimally invasive, small incision and fast recovery, and can obtain the same clinical effect as traditional open surgery (7). MED is based on traditional open surgery and microsurgery, and it is basically the same as that of open surgery (8). However, it has a similar clinical curative effect to traditional open surgery and has the advantages of minimally invasive surgery for patients (9). At present, it is believed that both PTED and MED can treat LDH safely and effectively, and both have their own characteristics. There are some advantages and disadvantages in the treatment of LDH, which makes it difficult for surgeons to choose. Some studies have shown that PETD can make the patients recover quickly and can achieve better clinical results after at least 2 years of follow-up under limited indications (10). However, some studies revealed that MED and PETD have the same effect on $\mathrm{LDH}$, but there is no significant difference (11).

Therefore, in the present study, we aimed to compare and analyze the application value of PTED and MED in LDH treatment to identify a more effective and safe surgical treatment for LDH patients.

\section{Materials and methods}

Research participants. One-way study was conducted in $108 \mathrm{LDH}$ patients who underwent surgical treatment in the 
Affiliated Hospital of North Sichuan Medical College from January 2017 to July 2018. The patients were then divided into PTED group (treated with PTED, $n=50$ ) and MED group (treated with MED, $n=58$ ). Inclusion criteria were: Patients were confirmed as LDH by imaging and clinical symptoms; 6-week conservative treatment was ineffective for patients; patients firstly underwent surgery; patients aged 18-65 years; patients could independently complete all evaluations of the study; patients were followed up for one year. Exclusion criteria: Patients in pregnancy; patients underwent spinal surgery before intervention; patients with contraindications to surgery; patients with serious systemic diseases; patients with incomplete clinical data.

This study was approved by the Ethics Committee of the Affiliated Hospital of North Sichuan Medical College, and all the subjects have signed informed consent forms.

Surgical treatment. In PTED group, $1 \%$ lidocaine was used for regional anesthesia for needle insertion, patients were placed in the prone position, the abdomen was suspended, and the waist was flexed. Mid-line of spinous process and iliac crest were accurately marked, local anesthesia was performed, a small incision of $0.8 \mathrm{~cm}$ was made by cutting at $10-13 \mathrm{~cm}$ near the mid-line of the surgical segment, and the puncture was performed under $\mathrm{C}$-arm fluoroscopy. After reaching the posterolateral side of the herniated nucleus pulposus, $1 \mathrm{ml}$ methylene blue was injected into the intervertebral disc to determine the damaged nucleus pulposus, the guide wire was inserted, and the puncture needle was withdrawn. A dilating tube was inserted along the guide wire, a working sleeve was placed under the nerve root, the guide wire was withdrawn and the tube was expanded, and then the soft tissue was expanded step by step. The foramen and camera were connected to determine the nucleus pulposus, dura mater and nerve root. The nucleus pulposus tissue was taken out by using a nucleus pulposus forceps. Bipolar radio frequency ablation was performed to stop bleeding, disc ablation decompression was performed, shrinkage and forming of a posterior longitudinal ligament and a fiber tear opening were then conducted. The operation was stopped after the disc tissue was removed completely and the nerve root was loosened satisfactorily. Finally, the working sleeve was withdrawn, and the wound was disinfected and sutured.

The MED group adopted epidural anesthesia, prone position, abdominal suspension, lumbar flexion, and C-wall fluoroscopy machine to locate the surgical gap. A longitudinal incision of $1.5 \mathrm{~cm}$ was cut on the lateral side of the spinous process. The skin, subcutaneous tissue and lumbar dorsal fascia were cut off and inserted with channel tube. The endoscope was inserted to observe the surgical fields. The lower edge of the vertebral plate was curetted, the bone and ligamentum flavum at the lower side of the vertebral plate were removed with a bone rongeur, the dural sac and nerve root were stretched with nerve pulling hook, the prolapsed or protruding nucleus pulposus were removed by nucleus pulposus forceps, and the nerve root canal was expanded. The conventional intervertebral foramen forming treatment was performed. After the nerve root was loosened satisfactorily, the wound was disinfected and treated to stop bleeding, the cannula was withdrawn, and the incision was sutured.
All operations were performed by surgeons who had received professional training from PTED and MED in our hospital. Both groups of patients received routine antibiotics after operation, avoiding strenuous exercise within 3 months after operation.

Outcome measures. The incision length, operation time, intraoperative fluoroscopic guidance times, intraoperative blood loss, postoperative bed rest time and length of hospital stay of the two groups were recorded, and the incidence rate of postoperative complications of the two groups was counted.

At 3 months after operation, the improvement of clinical symptoms of the two groups of patients was evaluated by the MacNab scale (12), which was divided into four parts: Excellent, good, general and bad, wherein the excellent rate $=($ excellent + good $) /$ total number $* 100 \%$.

VAS scale (13) was used to evaluate the postoperative pain degree of patients, with a total score of 10 points. A high score was closely related to the high pain intensity. Oswestry Disab ility Index (ODI) (14) was used to assess the improvement of patients' dysfunction. The scale included pain intensity, daily activity and social activity, with a total score of 45 points. A high score was closely related to the high degree of dysfunction.

The Japanese Orthopaedic Association Scale (JOA) (15) was used to assess the improvement of lumbar spine function, with a total score of 17 points. A high score was closely related to the better function recovery.

Quality of life questionnaire (QLQ) (16) was used to assess the patients' quality of life after 3 months of discharge, including general health $(\mathrm{GH})$, role-physical (RP), physical functioning $(\mathrm{PF})$, social function $(\mathrm{SF})$, emotional role (RE) and mental health $(\mathrm{MH})$, each with 100 points. A high score was closely related to the better quality of life.

Statistical analysis. SPSS 21.0 (IBM Corp.) was used for statistical analysis, and GraphPad Prism 7 (GraphPad Software, Inc.) for visualizing the data images. The Chi-square test or Fisher's exact test was used to compare the counting data in this study. Independent t-test was used for comparison of the measurement data, and repeated measurement data were compared by analysis of variance. LSD-t was used for subsequent analysis. Kaplan-Meier method was used to visualize the recurrence curve of patients, and the log-rank test was used for analysis. $\mathrm{P}<0.05$ indicated a statistical difference.

\section{Results}

Comparison of general data. There was no significant difference in sex, age, weight, work type and operative segment between the two groups $(\mathrm{P}>0.05)$ (Table I).

Comparison of surgical conditions. The operation was successfully completed in the two groups. Comparing the operation of the two groups of patients, it was found that the MED group was better than the PTED group in the number of intraoperative fluoroscopic guidance and operation time $(\mathrm{P}<0.05)$, while the PTED group was better than the MED group in the intraoperative blood loss, surgical incision length, length of hospital stay and bed rest time $(\mathrm{P}<0.05)$ (Table II). 
Table I. Comparison of general data of two groups of patients.

\begin{tabular}{|c|c|c|c|c|}
\hline Group & PTED group $(\mathrm{n}=50)$ & MED group $(n=58)$ & $\chi^{2} / \mathrm{t}$ & P-value \\
\hline Sex & & & 0.621 & 0.431 \\
\hline Female & $17(34.00)$ & $24(41.38)$ & & \\
\hline Male & $33(66.00)$ & $34(58.62)$ & & \\
\hline Average age (years) & $40.98 \pm 7.98$ & $42.38 \pm 8.11$ & 0.902 & 0.370 \\
\hline Average body weight (kg) & $63.21 \pm 6.33$ & $61.66 \pm 7.18$ & 1.181 & 0.240 \\
\hline Occupation & & & 0.225 & 0.635 \\
\hline Busy & $29(58.00)$ & $31(53.45)$ & & \\
\hline Relaxed & $21(42.00)$ & $27(46.55)$ & & \\
\hline Segments & & & 1.811 & 0.404 \\
\hline L3-4 & $3(6.00)$ & $5(8.62)$ & & \\
\hline L4-5 & $28(56.00)$ & $25(43.10)$ & & \\
\hline $\mathrm{L} 5 \sim \mathrm{S} 1$ & $19(38.00)$ & $28(48.28)$ & & \\
\hline
\end{tabular}

Table II. Comparison of surgical conditions between two groups.

\begin{tabular}{|c|c|c|c|c|}
\hline Group & PTED group $(n=50)$ & MED group $(n=58)$ & $\mathrm{t}$ & P-value \\
\hline Operation time (min) & $74.23 \pm 15.81$ & $49.56 \pm 12.56$ & 9.031 & $<0.001$ \\
\hline Length of stay (days) & $5.25 \pm 1.57$ & $7.19 \pm 1.85$ & 5.824 & $<0.001$ \\
\hline Intraoperative blood loss (ml) & $17.15 \pm 2.12$ & $24.86 \pm 3.15$ & 14.674 & $<0.001$ \\
\hline Bed rest time $(\mathrm{h})$ & $11.56 \pm 5.85$ & $62.95 \pm 6.56$ & 42.663 & $<0.001$ \\
\hline Length of surgical incision $(\mathrm{cm})$ & $0.75 \pm 0.15$ & $1.88 \pm 0.22$ & 30.680 & $<0.001$ \\
\hline Number of intraoperative fluoroscopy instructions & $19.56 \pm 9.56$ & $4.58 \pm 1.52$ & 11.771 & $<0.001$ \\
\hline
\end{tabular}

Table III. Improvement of clinical symptoms.

\begin{tabular}{|c|c|c|c|c|}
\hline Groups & PTED group $(n=50)$ & MED group $(n=58)$ & $\chi^{2}$ & P-value \\
\hline Excellent & $29(58.00)$ & $25(43.10)$ & 2.383 & 0.123 \\
\hline Good & $16(32.00)$ & $25(43.10)$ & 1.406 & 0.236 \\
\hline General & $4(8.00)$ & $6(10.34)$ & 0.176 & 0.675 \\
\hline Bad & $1(2.00)$ & $2(3.45)$ & 0.209 & 0.648 \\
\hline Satisfactory rate & $90.00 \%$ & $86.21 \%$ & 0.365 & 0.546 \\
\hline
\end{tabular}

Improvement of clinical symptoms. MacNab was used to evaluate the clinical efficacy of patients. PTED and MED had good therapeutic effects on LDH patients, and the therapeutic effects of the two groups were equivalent $(\mathrm{P}>0.05)$ (Table III).

Comparison of pain assessment. Evaluation of patients' pain showed that there was no statistical difference in the preoperative VAS scores between the two groups $(\mathrm{P}>0.05)$. The postoperative VAS scores of the two groups were decreased $(\mathrm{P}<0.05)$, but there was no statistical difference between the two groups $(\mathrm{P}>0.05)$ (Fig. 1).

Comparison of ODI and JOA scores of patients. ODI and JOA were used to evaluate the recovery of patients. We found that there was no statistical difference between the two groups in preoperative ODI and JOA scores $(\mathrm{P}>0.05)$. After operation, the ODI score decreased whereas the JOA score increased in the two groups $(\mathrm{P}<0.05)$, but there was no significant difference between the two groups $(\mathrm{P}>0.05)$ (Fig. 2).

Postoperative complications. After recording and comparing the postoperative complications of the two groups of patients, it was found that there were 2 cases of poor wound healing and 1 case of temporary sensory disturbance in PTED group. In MED group, there were 3 cases of poor wound healing, 2 cases of temporary sensory disturbance and 3 cases of nerve injury. There was no significant difference in complication rate between the two groups $(\mathrm{P}>0.05)$ (Table IV). 
Table IV. Incidence of postoperative complications.

\begin{tabular}{lccr}
\hline Groups & PTED group $(\mathrm{n}=50)$ & MED group (n=58) & $\chi^{2}$ \\
\hline Nerve injury & 0 & $3(5.17)$ & 0.103 \\
Poor wound healing & $2(4.00)$ & $3(5.17)$ & 2.660 \\
Temporary sensory disturbance & $1(2.00)$ & $2(3.45)$ & 0.289 \\
Total complication rate & $3(6.00)$ & $8(13.29)$ & 0.209 \\
\hline
\end{tabular}

Table V. Comparison of quality of life.

\begin{tabular}{|c|c|c|c|c|}
\hline Group & PTED group $(\mathrm{n}=50)$ & MED group $(n=58)$ & $\mathrm{t}$ & P-value \\
\hline $\mathrm{GH}$ & $74.64 \pm 6.23$ & $76.34 \pm 6.87$ & 1.338 & 0.184 \\
\hline $\mathrm{RP}$ & $77.23 \pm 5.45$ & $79.33 \pm 6.15$ & 1.864 & 0.065 \\
\hline $\mathrm{PF}$ & $76.61 \pm 5.97$ & $75.42 \pm 5.82$ & 1.047 & 0.298 \\
\hline SF & $74.12 \pm 4.87$ & $75.67 \pm 5.77$ & 1.495 & 0.138 \\
\hline RE & $79.42 \pm 5.22$ & $78.88 \pm 6.31$ & 0.480 & 0.632 \\
\hline $\mathrm{MH}$ & $81.76 \pm 5.98$ & $80.24 \pm 5.81$ & 1.337 & 0.184 \\
\hline
\end{tabular}

GH, general health; RP, role-physical; PF, physical functioning; SF, social function; RE, emotional role and MH, mental health.

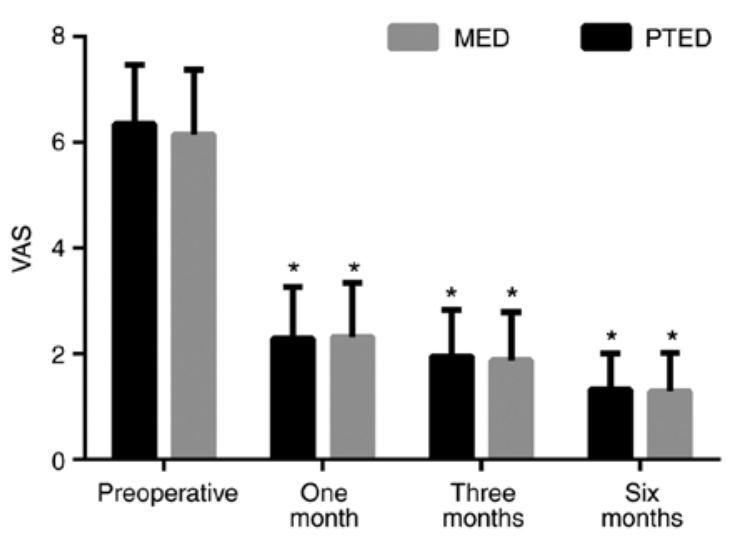

Figure 1. Comparison of VAS scores of two groups of patients at different time points. ${ }^{*} \mathrm{P}<0.05$ compareed with the preoperative in the same group.

Comparison of quality of life. Assessment of patient quality of life showed that there was no significant difference in GH, RP, $\mathrm{PF}, \mathrm{SF}, \mathrm{RE}$ and $\mathrm{MH}$ scores in QOL between the two groups $(\mathrm{P}>0.05)$ (Table V).

Comparison of postoperative recurrence. During the one-year follow-up, 4 patients $(8.00 \%)$ in PTED group relapsed, while 6 patients $(10.34 \%)$ in MED group relapsed. The recurrence curves of the two groups were visualized, and there was no significant difference between the two groups in 1 year recurrence (Log-rank: $\mathrm{P}=0.668$ ) (Fig. 3).

\section{Discussion}

LDH is the most common spinal disease in clinic. Patients often suffer from lumbago and leg pain, muscle weakness, limb numbness and other symptoms, which have a great negative impact on the life and work of patients (17). At present, surgery is a common method of LDH treatment, but traditional open surgery has many shortcomings, such as large incision, large intraoperative hemorrhage, slow postoperative recovery and many other complications (18). In nearly 10 years, spine minimally invasive surgery has developed rapidly, and LDH surgical treatment has developed from traditional open surgery to minimally invasive surgery (19). However, the prognosis of LDH patients varies with different operations. Therefore, finding a safer and more effective surgical method is one of the current research hotspots.

PTED and MED are two kinds of minimally invasive spinal surgery commonly used nowadays. Compared with traditional open surgery, both of them can ensure the clinical efficacy equivalent to traditional open surgery and have the advantages of minimally invasive surgery $(7,9)$. In the present study, we first analyzed the advantages and disadvantages of PTED and MED in perioperative period. The results showed that MED group was better than PTED group in the number of intraoperative fluoroscopic guidance and operation time, while PTED group was better than MED group in the intraoperative blood loss, surgical incision length, length of hospital stay and bed time. Recently, a retrospective study similar to our results showed that PTED had a significant advantage compared with MED in terms of incision length, intraoperative blood loss, bed rest time and length of hospital stay (20). The reason may be that PTED is based on endoscopic technology and percutaneous puncture technology and has less wound to the patient, thus being more conducive to postoperative recovery of the patient.

$\mathrm{MacNab}$ is a commonly used indicator to evaluate the improvement of symptoms of LDH patients (12). In this study, we used MacNab to evaluate the curative effect of the two 

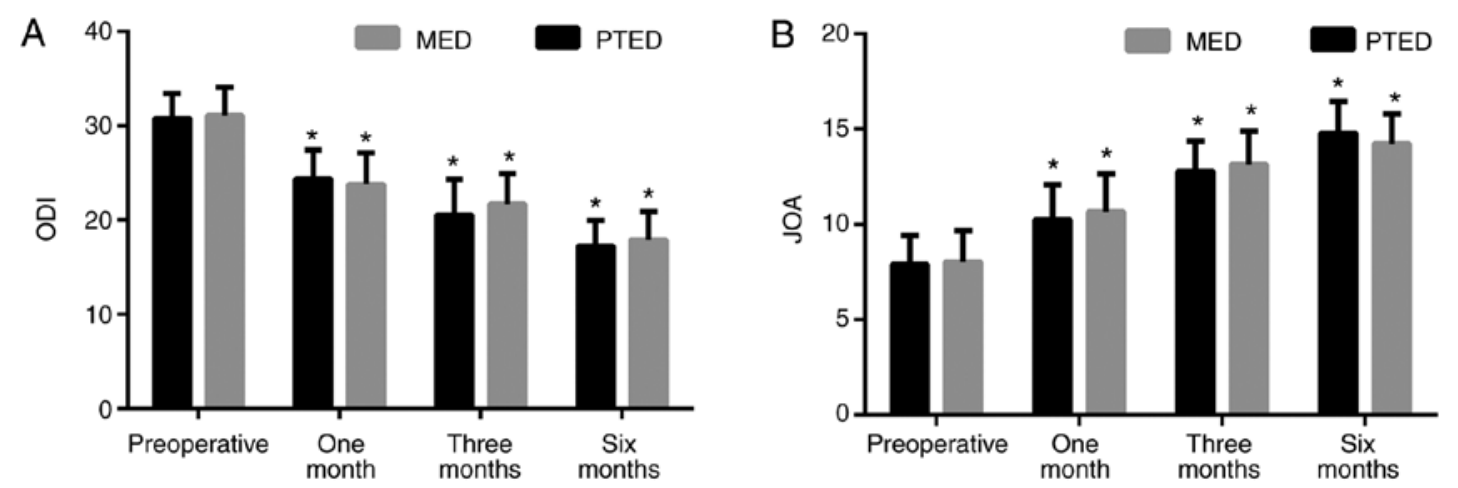

Figure 2. Comparison of ODI and JOA score of patients. (A) Comparison of ODI and (B) JOA scores between the two groups at different time points. "P<0.05 compareed with the preoperative in the same group.

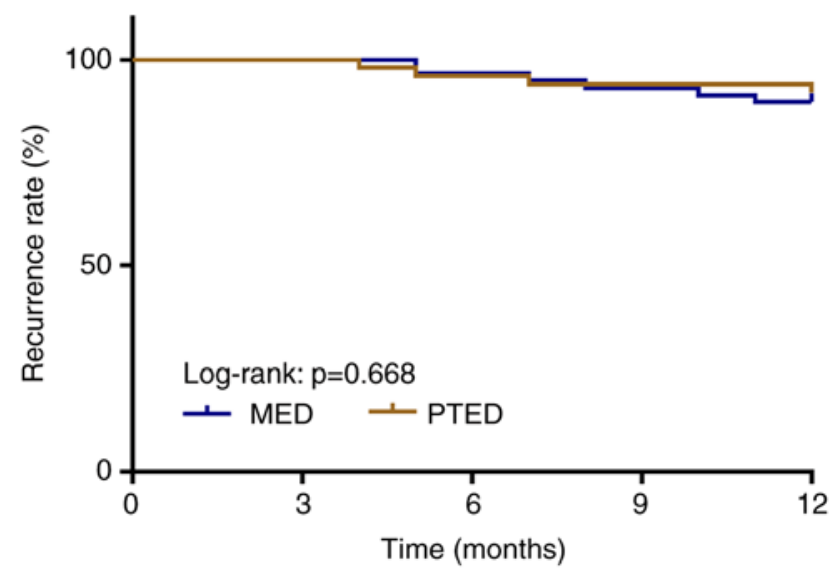

Figure 3. Comparison of postoperative recurrence between PTED and MED groups.

groups of patients, and found that the excellent rate of PTED group was $90.00 \%$, and that of MED group was $86.21 \%$. This showed that PTED and MED could improve the clinical symptoms of LDH patients, and the effects of the two are equivalent. Pain is the direct cause affecting the work and life of LDH patients. Therefore, relieving pain is the main goal of LDH treatment (21). ODI is one of the main outcome indicators widely used to evaluate patients with spinal diseases and is closely related to patient pain. JOA is a common index for evaluating lumbar spine function of patients. Our results showed that the ODI and VAS scores of the two groups of patients decreased and JOA scores increased, while there was no significant difference of the three scores between the two groups of patients. This showed that PTED and MED could effectively improve lumbago and leg pain of LDH patients and reduce the degree of lumbar dysfunction. In addition, quality of life results for the two groups of patients showed no significant difference. These findings indicated that PTED and MED have little difference in the effect of treating LDH, and they are both effective methods for treating LDH. Previous research results are similar to our results, demonstrating that PTED and MED have the same effect on LDH (22).

Although PTED and MED are both minimally invasive operations, there are still some complications. We recorded and compared the postoperative complications in PTED and MED groups, and found that there were no incurable complications in the two groups, and the complications of the two were similar. This confirmed that both were safe methods for LDH treatment. However, it is worth noting that there are no patients with nerve injury and damage in PTED group, while there are 3 patients in MED group. This result may be caused by a longer incision for patients in MED group. Recurrence is the main cause of reoperation for $\mathrm{LDH}$ patients. The recurrence rate of LDH patients was 5-15\% (23). During the one-year follow-up of patients, we found that 4 patients in PTED group had recurrence, while 6 patients in MED group had recurrence, with the recurrence rates ranging from 5 to $15 \%$.

This study made clear the advantages and disadvantages of PTED and MED in the treatment of LDH, which has certain clinical guiding value, but has certain limitations. First, the follow-up time was relatively short, and the long-term results of the two surgical methods for LDH patients were not clear. Secondly, the medical expenses used by the two groups of patients were not recorded. In addition, the therapeutic effects of PTED and MED on patients of different ages, sexes and prominent types have not been further explored. The number of subjects in this study is limited, and subsequent large-scale clinical studies are still needed.

To sum up, both PTED and MED can effectively treat LDH, and there are some advantages and disadvantages. MED is superior to PTED in the number of intraoperative fluoroscopy instructions and the operation time, while PTED is superior to MED in the amount of intraoperative hemorrhage, the length of surgical incision, the length of hospital stay and the bed time. In conclusion, we believe that PTED may be the first choice for LDH.

\section{Acknowledgements}

Not applicable.

\section{Funding}

The present study is funded by the Popularization Project of Health Research in Sichuan Province (19PJ202), China.

\section{Availability of data and materials}

The datasets used and/or analyzed during the present study are available from the corresponding author on reasonable request. 


\section{Authors' contributions}

YZ, JC, HX and NF led the conception and design of this study. YZ, KL, YW, QC, CJ and JH were responsible for the data collection and analysis. JC, HX, KL and YW were in charge of interpreting the data and drafting the manuscript. YZ, YW and QC made revision from critical perspective for important intellectual content. The final version was read and adopted by all the authors.

\section{Ethics approval and consent to participate}

The study was approved by the Ethics Committee of Affiliated Hospital of North Sichuan Medical College. Signed written informed consents were obtained from the patients.

\section{Patient consent for publication}

Not applicable.

\section{Competing interests}

The authors declare that they have no competing interests.

\section{References}

1. Amin RM, Andrade NS and Neuman BJ: Lumbar disc herniation. Curr Rev Musculoskelet Med 10: 507-516, 2017.

2. Hoy D, March L, Brooks P, Blyth F, Woolf A, Bain C, Williams G, Smith E, Vos T, Barendregt J, et al: The global burden of low back pain: Estimates from the Global burden of disease 2010 study. Ann Rheum Dis 73: 968-974, 2014.

3. Soman SM, Modi JV and Chokshi J: Feasibility of endoscopic discectomy by inter laminar approach at a high volume tertiary public hospital in a developing country. J Spine Surg 3: 38-43, 2017.

4. Telfeian AE, Veeravagu A, Oyelese AA and Gokaslan ZL: A brief history of endoscopic spine surgery. Neurosurg Focus 40 : E2, 2016.

5. Bhatia PS, Chhabra HS, Mohapatra B, Nanda A, Sangodimath G and Kaul R: Microdiscectomy or tubular discectomy: Is any of them a better option for management of lumbar disc prolapse. J Craniovertebr Junction Spine 7: 146-152, 2016.

6. Hirano Y, Mizuno J, Takeda M, Itoh Y, Matsuoka H and Watanabe K: Percutaneous endoscopic lumbar discectomy-early clinical experience. Neurol Med Chir (Tokyo) 52: 625-630, 2012.

7. Zhang Y, Pan Z, Yu Y, Zhang D, Ha Y, Yi S, Shin DA, Sun J, Koga H, Phan K, et al: The modified transforaminal endoscopic technique in treating intracanalicular combining foraminal and/or extraforaminal lumbar disc herniations. Quant Imaging Med Surg 8: 936-945, 2018.

8. Foley KT, Smith MM and Rampersaud YR: Microendoscopic approach to far-lateral lumbar disc herniation. Neurosurg Focus 7: e5, 1999.
9. Marappan K, Jothi R and Paul Raj S: Microendoscopic discectomy (MED) for lumbar disc herniation: Comparison of learning curve of the surgery and outcome with other established case studies. J Spine Surg 4: 630-637, 2018.

10. Liu X, Yuan S, Tian Y, Wang L, Gong L, Zheng Y and Li J: Comparison of percutaneous endoscopic transforaminal discectomy, microendoscopic discectomy, and microdiscectomy for symptomatic lumbar disc herniation: Minimum 2-year follow-up results. J Neurosurg Spine 28: 317-325, 2018.

11. Chen Z, Zhang L, Dong J, Xie P, Liu B, Wang Q, Chen R, Feng F, Yang B, Shu T, et al: Percutaneous transforaminal endoscopic discectomy compared with microendoscopic discectomy for lumbar disc herniation: 1-Year results of an ongoing randomized controlled trial. J Neurosurg Spine 28: 300-310, 2018.

12. Hirachan MP, Gao Z, Lin Y and Singh R: Clinical outcome of percutaneous endoscopic lumbar surgery (PELS) in treatment of lumbar disc herniation. Open J Orthop 7: 99-109, 2017.

13. Singh AP, Kohli V and Bajwa SJ: Intravenous analgesia with opioids versus femoral nerve block with $0.2 \%$ ropivacaine as preemptive analgesic for fracture femur: A randomized comparative study. Anesth Essays Res 10: 338-342, 2016.

14. Fairbank JC and Pynsent PB: The oswestry disability index. Spine (Phila Pa 1976) 25: 2940-2952, 2000.

15. Kopjar B, Tetreault L, Kalsi-Ryan S and Fehlings M: Psychometric properties of the modified Japanese orthopaedic association scale in patients with cervical spondylotic myelopathy. Spine (Phila $\mathrm{Pa}$ 1976) 40: E23-E28, 2015

16. Arcopinto M, Salzano A, Giallauria F, Bossone E, Isgaard J, Marra AM, Bobbio E, Vriz O, Åberg DN, Masarone D, et al: Growth hormone deficiency is associated with worse cardiac function, physical performance, and outcome in chronic heart failure: Insights from the T.O.S.CA. GHD study. PLoS One 12: e0170058, 2017.

17. Leung VY, Aladin DM, Lv F, Tam V, Sun Y, Lau RY, Hung SC, Ngan AH, Tang B, Lim CT, et al: Mesenchymal stem cells reduce intervertebral disc fibrosis and facilitate repair. Stem Cells 32: 2164-2177, 2014.

18. Bai J, Zhang W, Liu X, Cheng J, Wang X, Ding W and Shen Y: Percutaneous transforaminal endoscopic discectomy in the treatment of senior patients with lumbar degenerative disc disease. Exp Ther Med 17: 874-882, 2019.

19. Kosztowski TA, Choi D, Fridley J, Galgano M, Gokaslan Z, Oyelese A and Telfeian AE: Lumbar disc reherniation after transforaminal lumbar endoscopic discectomy. Ann Transl Med 6: 106, 2018.

20. Wang F, Guo D, Sun T and Guan K: A comparative study on short-term therapeutic effects of percutaneous transforaminal endoscopic discectomy and microendoscopic discectomy on lumbar disc herniation. Pak J Med Sci 35: 426-431, 2019.

21. Klineberg E, Ching A, Mundis G, Burton D and Bess S: Diagnosis, treatment, and complications of adult lumbar disk herniation: Evidence-based data for the healthcare professional. Instr Course Lect 64: 405-416, 2015.

22. Sinkemani A, Hong X, Gao ZX, Zhuang SY, Jiang ZL, Zhang SD, Bao JP, Zhu L, Zhang P, Xie XH, et al: Outcomes of microendoscopic discectomy and percutaneous transforaminal endoscopic discectomy for the treatment of lumbar disc herniation: A comparative retrospective study. Asian Spine J 9: 833-840, 2015.

23. Huang W, Han Z, Liu J, Yu L and Yu X: Risk factors for recurrent lumbar disc herniation: A systematic review and meta-analysis. Medicine (Baltimore) 95: e2378, 2016. 